\title{
Sistema POLCA: revisão, classificação e análise da literatura
}

\section{POLCA System: literature review, classification, and analysis}

\author{
Fernanda Silva Chinet ${ }^{1}$ \\ Moacir Godinho Filho'
}

\begin{abstract}
Resumo: Este trabalho apresenta uma revisão bibliográfica (20 artigos) sobre o sistema de coordenação de ordem (SCO) denominado Paired-cell Overlapping Loops of Cards with Authorization (POLCA). A partir de tal revisão, propôs-se um sistema de classificação do sistema POLCA baseado em quatro categorias: sistema POLCA considerado (original/modificado); objetivo principal da pesquisa; fonte da publicação; e metodologia de pesquisa empregada. Uma vez classificada e estruturada, a revisão bibliográfica serviu de base para uma análise do tema. A literatura pesquisada mostrou a existência de algumas lacunas que merecem ser exploradas em pesquisas futuras, são elas: proposta de novas melhorias no sistema POLCA original, ou mesmo a hibridização de tal sistema com outro SCO; comparação, via simulação ou teoria de filas, do sistema POLCA com outros SCOs utilizados em ambientes de alta variedade de produtos; mostrar a aplicação do sistema POLCA na prática. Além disso, referente à prática, principalmente brasileira, tem-se que o sistema POLCA ainda é pouco conhecido em território nacional. Devido a esse fato, muitas oportunidades existem para os gerentes de Planejamento e Controle da Produção na prática, uma vez que internacionalmente tal sistema vem mostrando excelentes resultados em termos de redução de lead time.
\end{abstract}

Palavras-chave: POLCA. Planejamento e Controle da Produção. Revisão bibliográfica.

\begin{abstract}
This paper presents a literature review (20 articles) on the system for coordinating order (SCO) called Paired-cell Overlapping Loops of Cards with Authorization (POLCA). Based on this review, a system for classifying the POLCA was proposed including four dimensions: the POLCA system (original and modified), main purpose of research, paper source, and research method used. Once classified and organized, the literature review provided a basis for a comprehensive topic analysis. The review conducted identified some gaps in the literature that should be explored in future studies, such as: improvements to the original POLCA system or hybridization of this system with other SCOs; comparison by simulation or queuing models of the POLCA system with other SCOs used in high product variety environments; and a practical application of the POLCA system. Furthermore, with regard to its use, especially in Brazil, it was found that POLCA is still little known nationally.
\end{abstract}

Keywords: POLCA system. Production Planning and Control. Literature review.

\section{Introdução}

De acordo com Fernandes e Godinho Filho (2010), as atividades do Planejamento e Controle da Produção (PCP) envolvem uma série de decisões com o objetivo de definir o que, quanto e quando produzir, comprar e entregar, além de quem e/ou como produzir. Algumas das principais atividades do PCP, de acordo com esses autores são: previsão de demanda, planejamento agregado, programa mestre de produção, coordenação de ordens, programação da produção e controle de estoques.

O foco do presente trabalho é na atividade de coordenação de ordens. Um Sistema de Coordenação de Ordens (SCO), de acordo com Fernandes e Godinho Filho (2007), programa ou organiza/explode as necessidades em termos de componentes e materiais, e/ou controla a emissão/liberação das ordens de produção e compra, e/ou programa/sequencia as tarefas nas máquinas. Portanto, um SCO coordena as ordens de produção e de compra no chão de fábrica. Mais especificamente, o presente trabalho apresenta uma revisão da literatura sobre o SCO denominado POLCA (Paired-cell Overlapping Loops of Cards with Authorization), proposto por Suri (1998). Este sistema é parte de uma estratégia maior proposta pelo autor denominada Quick Response Manufacturing - QRM (SURI, 1998, 2010).

Apesar de ter sido proposto há mais de dez anos, a literatura sobre o sistema POLCA ainda é escassa, principalmente no Brasil. Diante disso, o objetivo do presente trabalho é realizar uma revisão da literatura

${ }^{1}$ Departamento de Engenharia de Produção, Universidade Federal de São Carlos - UFSCar, Rod. Washington Luís, Km 235 ,

CEP 13565-905, São Carlos, SP, e-mail: fernandachinet@yahoo.com.br; moacir@dep.ufscar.br

Recebido em 2/11/2011 — Aceito em 4/9/2012

Suporte financeiro: Nenhum. 
a respeito do sistema POLCA. Além disso, o presente trabalho, a partir de tal revisão, também propõe um sistema de classificação, a qual permite melhor análise da literatura e proposta de pesquisas futuras a respeito do tema em questão. A contribuição esperada é maior conhecimento e divulgação a respeito dos estudos existentes atualmente sobre o sistema POLCA. Para se obter um conhecimento geral sobre um determinado assunto ou área, a realização de uma classificação é uma ferramenta essencial, uma vez que a classificação é a base do conhecimento científico (GOOD, 1965).

Este trabalho apresenta-se estruturado da seguinte forma: na seção 2, são apresentados conceitos fundamentais sobre o POLCA; na seção 3, é mostrado o sistema de classificação proposto, bem como sua utilização para a classificação e codificação dos trabalhos encontrados na revisão bibliográfica efetuada; na seção 4, a revisão da literatura é estruturada; na seção 5, são realizadas análises sobre o tema; e, finalmente, na seção 6 , são apresentadas algumas conclusões.

\section{Sistema POLCA}

Suri (1998) define o sistema POLCA como uma estratégia híbrida que puxa e empurra a produção, combinando características dos sistemas Material Requirement Planning - MRP (empurrado) e Kanban (puxado).

De acordo com Fernandes e Godinho Filho (2007), o sistema POLCA apresenta basicamente quatro características:

(i) Autorização de liberação por meio de um sistema denominado HL/MRP (higher level MRP). O sistema HL/MRP é semelhante ao sistema MRP convencional com duas diferenças: a) o HL/MRP é baseado em uma estrutura de produtos simplificada, utilizando os lead times das células ao invés dos lead times individuais dos centros de trabalho dentro de cada célula; e b) as datas planejadas pelo HL/MRP são apenas datas nas quais as tarefas podem ser iniciadas (por isso são chamadas datas de autorização e não de liberação como no MRP convencional), sendo que seu início concreto na produção se dá somente mediante esta autorização, e também mediante a disponibilidade do cartão POLCA na célula que vai iniciar o trabalho.

(ii) Método de controle de material baseado em um cartão (denominado cartão POLCA). Estes cartões são utilizados para comunicação e controle entre as células; dentro das células pode ser usado outro sistema de controle, como o Kanban.

(iii) Os cartões POLCA, ao invés de se referirem especificamente a um produto, se referem a um par de células escolhidas da seguinte forma: se o roteiro de uma ordem qualquer sair de uma célula $A$ para uma célula $\mathrm{B}$, então é criado um cartão POLCA A/B e assim por diante para as outras etapas do processamento.
Este procedimento de trabalhar com as células em pares faz com que o cartão POLCA garanta que uma célula somente irá trabalhar em uma tarefa para a qual a célula de destino tem capacidade disponível.

(iv) O cartão POLCA para cada par de células permanece com a tarefa durante toda sua jornada (incluindo processamento) através das duas células e depois retorna para a primeira célula quando é finalizado o processamento na segunda célula. Neste momento, a primeira célula pode iniciar outra tarefa.

Resumidamente, o funcionamento do sistema POLCA é o seguinte: quando uma empresa recebe um pedido de um cliente, o sistema HL/MRP usa os lead times planejados de cada célula para determinar quando cada célula no roteiro do produto pode iniciar o processamento da tarefa. Estas datas de autorização serão seguidas somente se um cartão POLCA estiver disponível na célula que inicia a operação.

Para melhor compreensão deste sistema, Suri (1998) e Suri e Krishnamurthy (2003) consideram o exemplo de uma empresa que fabrica placas de identificação personalizadas para aparelhos de pequeno e grande porte, denominada CFP Corporation. A empresa é dividida em quatro tipos de células (impressão, fabricação, montagem e expedição) para atender a seus mercados altamente variados por meio de uma estratégia QRM.

A fabricação das placas se inicia quando as três condições necessárias do sistema POLCA são atendidas: a produção é autorizada pelo sistema HL/ MRP, existe matéria-prima e existe um cartão POLCA.

A Figura 1 mostra os fluxos de um cartão POLCA para uma determinada ordem na CFP Corporation. $\mathrm{O}$ roteiro desta ordem se inicia de $\mathrm{P} 1$ para $\mathrm{F} 2$, segue para a montagem A4 e, posteriormente, para S1, para assim ser expedido. Esta ordem, portanto, prossegue através dos loops do cartão POLCA com os pares P1/F2, F2/A4 e A4/S1, como mostrado na Figura 1.

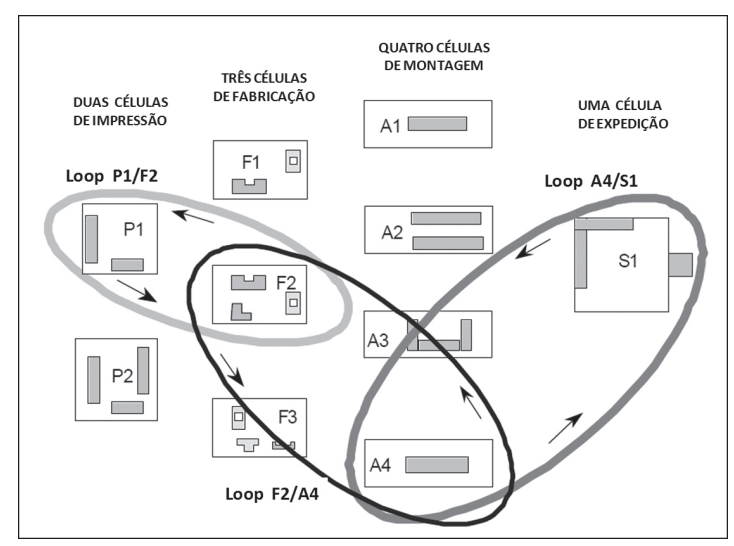

Figura 1. Fluxo do cartão POLCA para uma determinada ordem de produção na CPF Corporation. 
A ordem ilustrada é programada da seguinte forma. Com matéria-prima disponível em P1 e cartão POLCA P1/F2, a tarefa é lançada em P1. Depois de ter completado as operações nesta célula, a tarefa e o cartão POLCA P1/F2 seguem para o pulmão de entrada de F2. Quando um dos cartões F2/A4 chega de volta a F2, a tarefa é lançada dentro da célula F2. Neste momento, a tarefa em F2 carrega dois tipos de cartão POLCA, P1/F2 e F2/A4. Quando esta tarefa é completada, o cartão P1/F2 é devolvido para o início de $\mathrm{P} 1$ e a tarefa é encaminhada para o pulmão de entrada de A4 junto do cartão F2/A4. O mesmo processo se repete para a célula de montagem A4, na qual é necessário um cartão A4/S1 disponível para iniciar a tarefa na célula. Quando a tarefa é completada, esta segue para o pulmão de entrada de S1, enquanto o cartão F2/A4 volta para o início da célula $\mathrm{F} 2$. Como $\mathrm{S} 1$ é a última célula do roteiro, não haverá um cartão POLCA esperando para iniciar a produção e a tarefa pode ser lançada em S1 toda vez que a célula estiver preparada para iniciar outra tarefa. Quando a tarefa em S1 é completada, ela é expedida e o cartão A4/S1 retorna para o início de A4, concluindo assim a jornada de cartões POLCA para esta ordem de produção.

O sistema POLCA é atualmente utilizado com sucesso na prática em inúmeras empresas americanas e europeias (SURI, 2010). Entre estes casos, foram desenvolvidos trabalhos com o sistema POLCA adaptado às condições da empresa. Este sistema foi denominado POLCA modificado (PM) já que apresenta algumas modificações em relação ao sistema originalmente proposto por Suri (1998), o POLCA original (PO).

\section{Proposta de classificação da literatura sobre o sistema POLCA}

\subsection{Metodologia de pesquisa}

Segundo Rowley e Slack (2004), uma revisão bibliográfica sumariza o estado da arte do assunto em questão, apoiando a identificação do tópico de pesquisa, questão ou hipótese. Dessa forma, a revisão contribui para fornecer uma contextualização de um tema de pesquisa dentro de um universo de trabalhos já existentes na área, construindo e entendendo conceitos teóricos e terminologias.

A presente pesquisa foi realizada em cinco bases de dados, a saber: Compendex, Google Acadêmico, Web of Science, Scopus e Scielo. A revisão procurou ser exaustiva, de forma a abranger toda a literatura atualmente existente sobre o assunto. A palavra-chave utilizada foi "POLCA". Além das bases de dados internacionais, foram pesquisados dois periódicos nacionais, a revista Gestão e Produção e a revista
Produção, e os anais do Encontro Nacional de Engenharia de Produção (ENEGEP) e do Simpósio de Engenharia de Produção (SIMPEP). Foram incluídos somente livros, artigos publicados em periódicos (journals) e congressos, pois, de acordo com Ngai et al. (2008), são as fontes mais utilizadas para busca de informações e definição de pesquisas futuras. A partir disso, por meio da leitura dos resumos, 20 artigos foram selecionados para análise final.

Depois da identificação e análise dos artigos, foi proposto um sistema de classificação, detalhado na subseção 3.2. Os artigos selecionados foram então classificados (seção 3.3). Depois disso, os pontos mais importantes dos artigos são apresentados de acordo com o sistema de classificação proposto (seção 4), propiciando uma análise do tema e sugestões de pesquisas futuras (seções 5 e 6 ).

\subsection{O sistema de classificação proposto}

Nesta seção, é apresentado o sistema de classificação proposto após a realização de uma revisão da literatura sobre o sistema POLCA. O sistema de classificação está baseado em quatro categorias: i) sistema POLCA considerado (original/ modificado); ii) objetivo principal da pesquisa; iii) fonte da publicação; e iv) metodologia de pesquisa empregada. Para facilitar a classificação dos trabalhos, será feita uma codificação para cada atributo que as categorias possam assumir.

A primeira categoria do método de classificação proposto é o tipo de sistema POLCA considerado. A proposta de tal categoria deve-se ao fato de que atualmente estão surgindo novas versões e variações do POLCA na literatura. Dessa forma, essa categoria é dividida em duas classes: POLCA original (PO), que corresponde ao sistema originalmente proposto por Suri (1998) e mostrado na seção 2; e POLCA modificado (PM), que engloba sistemas com características do POLCA, mas que apresentam algumas modificações em relação ao sistema original.

Quanto à segunda categoria, tem-se que os trabalhos estudados podem ter como objetivo principal: (1) apresentar o funcionamento do sistema POLCA; (2) comparar qualitativamente o POLCA a outros SCOs; (3) comparar quantitativamente o POLCA a outros SCOs; (4) simular a aplicação do sistema POLCA; (5) mostrar aplicação na prática do sistema POLCA; e (6) somente citar o sistema POLCA como alternativa para o controle da produção de um sistema produtivo.

A terceira categoria do sistema de classificação refere-se à fonte de publicação dos trabalhos. Essa categoria é dividida em: livro (L); congresso local (CL); congresso internacional (CI); congresso nacional $(\mathrm{CN})$; e periódico internacional (PI).

Por fim, a quarta categoria está relacionada à metodologia de pesquisa empregada nos trabalhos. 
Depois de uma análise dos tipos de procedimentos de pesquisa utilizados nos trabalhos sobre o tema, quatro classes surgiram, definidas a seguir: (i) teórico conceitual (TC); (ii) estudo de caso (EC); (iii) simulação (S); e (iv) modelo de filas (QM).

De acordo com a tipologia empregada por Filippini (1997), Berto e Nakano (2000) e Nakano (2010), as três primeiras metodologias de pesquisa são conceituadas da seguinte forma: teórico conceitual são discussões conceituais a partir da literatura, revisões bibliográficas e modelagens conceituais; estudo de caso é uma análise aprofundada de um ou mais objetos (casos), com o uso de múltiplos instrumentos de coleta de dados e presença da interação entre pesquisador e objeto de pesquisa; e simulação faz o uso de técnicas computacionais para simular o funcionamento de sistemas produtivos a partir de modelos matemáticos. Já modelo de filas é definido por Shimizu (1984) como modelos matemáticos que consistem em programar corretamente as chegadas ou proporcionar o número suficiente de estações de serviço, de modo a diminuir o tempo de espera nas filas (bancos, supermercados, hospitais, ônibus). No caso de trabalhos sobre o POLCA, modelos de filas são utilizados basicamente como forma de modelar e avaliar o desempenho do sistema em questão.

\subsection{Classificação da revisão bibliográfica}

A revisão da literatura sobre o sistema POLCA foi composta por 20 trabalhos. A Tabela 1 mostra a classificação destes trabalhos quanto às quatro categorias propostas na seção anterior. Os artigos estão em ordem cronológica e alfabética dentro do ano de publicação.

\section{Revisão da literatura sobre sistema POLCA}

Nesta seção, a revisão bibliográfica é estruturada utilizando-se a classificação do tipo de sistema POLCA considerado (original/modificado), sendo que, dentro de cada uma dessas classes, os trabalhos foram agregados de acordo com seu(s) objetivo(s) principal(is). Um breve resumo de cada trabalho é apresentado, tentando ilustrar as principais contribuições para a literatura sobre o tema.

\subsection{Trabalhos que consideram o sistema POLCA original}

Dezessete artigos da presente revisão referem-se ao sistema POLCA original. Desses, apenas um artigo apresenta o funcionamento do sistema

Tabela 1. Classificação dos artigos da revisão bibliográfica sobre o sistema POLCA.

\begin{tabular}{lcccc}
\hline \multicolumn{1}{c}{ Artigo/ano de publicação } & $\begin{array}{c}\text { Classificação } \\
\text { quanto ao } \\
\text { sistema } \\
\text { POLCA } \\
\text { considerado }\end{array}$ & $\begin{array}{c}\text { Classificação } \\
\text { quanto ao } \\
\text { objetivo } \\
\text { principal da } \\
\text { pesquisa }\end{array}$ & $\begin{array}{c}\text { Classificação } \\
\text { quanto à fonte } \\
\text { de publicação }\end{array}$ & $\begin{array}{c}\text { Classificação } \\
\text { quanto à } \\
\text { metodologia } \\
\text { de pesquisa } \\
\text { empregada }\end{array}$ \\
\hline Suri (1998) & PO & 1 & L & TC \\
Krishnamurthy, Suri e Vernon (2000) & PO & 3 & CL & QM \\
Lödding, Yu e Wiendahl (2003) & PO & 3 & PI & S \\
Ryan e Choobineh (2003) & PO & 6 & PI & - (*) $^{-1,2}$ \\
Suri (2003) & PO & 1,2 & CL & TC \\
Suri e Krishnamurthy (2003) & PO & $1,2,5$ & CL & TC, EC \\
Krishnamurthy, Suri e Vernon (2004) & PO & 6 & PI & - (*) $^{-}$ \\
Vandaele et al. (2004) & PM & 1,5 & CL & TC, EC \\
Fernandes, Godinho Filho e Fonseca (2005) & PO & 2 & CN & TC \\
Stevenson, Hendry e Kingsman (2005) & PO & 2 & PI & TC \\
Suri (2005) & PO & 1,2 & PI & TC \\
Fernandes e do Carmo-Silva (2006) & PM & 1,3 & PI & TC, S \\
Riezebos (2006) & PO & 4 & CL & S \\
Baysan, Kabadurmus e Durmusoglu (2007) & PO & 4 & CI & S \\
Germs e Riezebos (2008) & PO & 3 & CL & S \\
Vandaele et al. (2008) & PM & 1,5 & PI & TC, EC \\
Kabadurmus (2009) & PO & 3 & CL & S \\
Krishnamurthy e Suri (2009) & PO & 1,5 & PI & TC, EC \\
Riezebos (2010) & PO & 1,5 & PI & TC, EC \\
Harrod e Kanet (2013) & PO & 4 & PI & S \\
\hline
\end{tabular}

(*) O traço indica que a metodologia do trabalho não foi analisada, uma vez que tal trabalho somente cita o POLCA (categoria 6). 
(seção 4.1.1); dois comparam qualitativamente o POLCA com outros SCOs (seção 4.1.2); quatro comparam quantitativamente o POLCA com outros SCOs (seção 4.1.3); três mostram uma simulação na prática do sistema POLCA (seção 4.1.4); e, finalmente, cinco apresentam mais de um objetivo principal (seção 4.1.4).

\subsubsection{Trabalhos que apresentam o funcionamento do sistema POLCA}

O sistema POLCA foi introduzido por Suri (1998) como um dos princípios do QRM para ser utilizado no controle da produção. $\mathrm{O}$ autor define POLCA como um sistema híbrido que combina os melhores fatores dos sistemas MRP (empurrado) e Kanban (puxado). Ainda de acordo com esse autor, o sistema supera algumas limitações do Kanban proporcionando alto grau de flexibilidade e permitindo a fabricação de produtos customizados. Em Suri (1998), o POLCA é introduzido por meio da explicação dos princípios fundamentais de seu funcionamento e exemplo de aplicação na prática.

\subsubsection{Trabalhos que comparam qualitativamente o POLCA com outros SCO}

Fernandes, Godinho Filho e Fonseca (2005) apresentam a lógica de funcionamento dos seguintes sistemas de coordenação de ordem: Descentralized Work in Process (DEWIP), Load Oriented Order Release (LOOR) e o POLCA. Com isso, foi possível identificar as características dos ambientes propícios à utilização destes sistemas e realizar uma classificação de acordo com estas características. Os autores concluíram que os três sistemas têm como variável de controle primária o Work in Process (WIP), sendo que os sistemas DEWIP e POLCA podem ser caracterizados como sistemas descentralizados e o sistema LOOR como centralizado. Além disso, são sistemas direcionados a ambientes com alta variedade e alta complexidade no fluxo de materiais e servem como uma alternativa ao sistema MRP, também adequado a este tipo de ambiente. Já Stevenson, Hendry e Kingsman (2005) mostram uma comparação de algumas abordagens clássicas utilizadas no Planejamento e Controle da Produção (PCP) tais como Kanban, Manufacturing Resource Planning (MRP II), Theory of Constraints (TOC) e algumas técnicas emergentes como Workload Control (WLC), Constant Work in Process (CONWIP) e POLCA. Dessa forma, foi possível fazer uma avaliação crítica dos métodos e suas aplicabilidades do ponto de vista de diversos setores da indústria make-to-order (MTO), a fim de auxiliar os praticantes na escolha de abordagens para o PCP. Segundo os autores, o WLC foi o sistema com maior destaque em ambientes job shop; enquanto que para outros ambientes, há várias alternativas, dependendo das características individuais e os objetivos da empresa. Eles também consideram que o sistema POLCA é uma abordagem muito recente e necessita de maior quantidade de pesquisas para confirmar sua aplicabilidade e desempenho em ambientes MTO.

\subsubsection{Trabalhos que comparam quantitativamente POLCA com outros SCOs}

Krishnamurthy, Suri e Vernon (2000) propõem uma nova abordagem baseada em modelos de filas para analisar sistemas de coordenação de ordens, tais como Kanban, CONWIP e POLCA. Tal abordagem, baseada na técnica de decomposição de nós, foi utilizada inicialmente para analisar um sistema Kanban estágio único e, depois, foi estendida para analisar sistemas Kanban de múltiplos estágios e sistemas CONWIP e POLCA com múltiplos produtos. Com isso, os autores mostraram que a ampliação desta abordagem para sistemas mais complexos tem como vantagem a diminuição da complexidade computacional. Nos casos de sistemas com múltiplos produtos, os cálculos não crescem com o aumento das redes decompostas e, para os casos de sistemas de múltiplos estágios, o rendimento de todas as fases dos estágios em série é conservado. Além disso, os autores mostraram quantitativamente a eficácia do POLCA quando comparado ao sistema Kanban. Lödding, Yu e Wiendahl (2003) apresentam uma comparação entre o sistema POLCA e outros SCOs (DEWIP, LOOR, CONWIP) em um ambiente job shop, por meio de uma simulação com dados reais da indústria. Os resultados mostraram que o LOOR foi o sistema que alcançou os melhores resultados com relação à redução de lead times e WIP. O DEWIP e o CONWIP tiveram aproximadamente o mesmo desempenho, porém o DEWIP tem uma aplicação muito mais ampla que o CONWIP. O POLCA não se mostrou adequado para o ambiente estudado, uma vez que, conforme citado pelos próprios autores, a sua aplicação requer que antes a manufatura celular seja utilizada, visando a redução da complexidade no fluxo de materiais, o que não foi realizado no estudo. Já Germs e Riezebos (2008) apresentaram em uma comparação entre SCOs que podem atuar em ambientes de alta variedade e customização. Dessa forma, a simulação foi utilizada para investigar a capacidade de balanceamento de carga de três sistemas: POLCA, CONWIP e m-CONWIP (sistemas CONWIP com vários loops operados simultaneamente). Com as comparações, foi possível analisar como as lógicas dos 3 sistemas influenciam na capacidade de balanceamento de carga e testar a influência das condições de produção sobre 
a capacidade de carga de trabalho destes sistemas. Os resultados das simulações confirmam a importância do balanceamento de carga de trabalho para melhor desempenho nos tempos de atravessamento total no chão de fábrica, conforme as condições de produção e o sistema utilizado. Além disso, o trabalho mostrou que o balanceamento de capacidade de carga pode ser aplicado para POLCA e m-CONWIP, porém não para o CONWIP. Por fim, Kabadurmus (2009) compara por meio de simulação, os sistemas POLCA e CONWIP em condições de demanda com grande instabilidade. Para um sistema hipotético, os autores criaram cenários com diferentes valores de variabilidade da demanda, variação do produto, variabilidade do tempo de processamento, tamanho de lote e taxa de inatividade. Os principais critérios de desempenho utilizados foram o lead time e WIP. Para os cenários estudados, o sistema POLCA superou o CONWIP, uma vez que o CONWIP não se adaptou bem em certos ambientes com demanda instável, máquinas com muitas falhas e alta variabilidade no processo.

\subsubsection{Trabalhos que mostram simulação na prática do sistema POLCA}

Neste grupo, o trabalho de Riezebos (2006) avalia a contribuição do sistema POLCA em uma empresa por meio de uma simulação de eventos discretos em um sistema de fluxo unidirecional. Para isto, foram realizados experimentos com modelos de ordem de chegada, tamanho do lote, variação de demanda, mix de produtos, ordem de prioridade e a ocorrência de quebras, a fim de mostrar o impacto do número de cartões POLCA sobre o tempo de atravessamento em um sistema de produção. Os resultados mostram que a quantidade de estoque em processo (WIP) está diretamente relacionada com o lead time e ambos decrescem se o número de cartões POLCA for reduzido. Desta forma, conclui-se que uma empresa que utiliza o sistema POLCA tem tendência de tornar-se mais flexível e capacitada para oferecer melhor serviço a seus clientes por apresentar um tempo total de atravessamento reduzido e controlado. Baysan, Kabadurmus e Durmusoglu (2007) ilustram uma análise econômica do sistema POLCA por meio da simulação de um sistema de manufatura real. A simulação é realizada em uma empresa de molde de vidros, em um ambiente engineering-to-order (ETO) com condições de demanda instável e uma grande variedade de produtos fabricados em pequenos lotes. O procedimento se deu pela implementação de uma estratégia de controle de materiais que exigiu um estudo de viabilidade de custos de estoque. Os resultados mostraram que, com a aplicação do POLCA, é possível obter uma redução dos WIP e, consequentemente, uma redução nos lead times e nos custos dos produtos da empresa. Já Harrod e
Kanet (2013) têm como objetivo estudar o controle do fluxo de trabalho em um ambiente make-to-order job shop considerando os resultados do cruzamento de dados de métodos de controle do fluxo de trabalho e modelos de filas de cada centro de trabalho. Para isto, foi realizada uma simulação de eventos discretos avaliando: a) cinco centros de trabalhos independentes com sequência e tempo de processamento selecionados randomicamente de uma matriz experimental com quatro tipos de controle do fluxo de trabalho (não controlada, Kanban, CONWIP, POLCA); e b) três modelos de filas (primeiro a chegar primeiro a atender, menor tempo de processamento, operação com prazo de entrega mais cedo). Os resultados mostraram que enquanto o estoque dos shops (ordens parcialmente completadas) é reduzido por meio dos métodos de controle do fluxo de trabalho, o estoque total das ordens de produtos acabados que estão pendentes, incompletas e interrompidas aumentaram. Além disso, conclui-se que a escolha do modelo de fila é mais significativa do que a escolha do método de controle do fluxo de trabalho.

\subsubsection{Trabalhos que possuem mais de um objetivo principal}

Esta categoria inclui os trabalhos que tratam de dois ou mais objetivos principais de forma conjunta. Dessa forma, três combinações de objetivos foram identificadas.

A primeira refere-se aos trabalhos que apresentam o funcionamento do sistema POLCA e também realizam uma comparação qualitativa com outros SCOs. Nessa linha, dois trabalhos foram encontrados. Suri (2003) apresenta o QRM e o sistema POLCA como uma combinação para as empresas do século XXI que produzem uma grande variedade de produtos com demanda altamente variável. O trabalho faz uma revisão geral da estratégia do QRM que foca na redução do lead time em toda a empresa e, explica por que as estratégias de fluxo unitário, takt time e produção puxada advindas do Lean Manufacturing não funcionam bem para mercados com alta variedade, propondo o QRM como estratégia adequada para esses ambientes. Além disso, é apresentada uma descrição do POLCA seguido de uma comparação qualitativa com o sistema Kanban. Por fim, conclui-se que a combinação de QRM e POLCA vem oferecer às empresas uma significativa vantagem competitiva por meio da capacidade de entregar produtos personalizados com curtos lead times. Já Suri (2005) apresenta o POLCA como uma estratégia enxuta para ambientes job shop. O POLCA é proposto como uma alternativa ao Kanban aplicado a produtos customizados ou de alta variedade, uma vez que este método torna-se ineficiente por causa 
da variedade de produtos sendo produzidos e da imprevisibilidade de volume requerido para cada peça. Desta forma, o trabalho apresenta o sistema POLCA como parte da estratégia QRM, em que explica seu funcionamento, os benefícios alcançados com sua implementação e as diferenças entre ele e o Kanban. Por fim, a autor conclui que o POLCA, quando combinado com outras estratégias QRM proporciona flexibilidade para absorver variações na demanda e no mix de produtos.

O segundo grupo de trabalhos que apresenta uma combinação de objetivos referentes ao POLCA original é formado por dois artigos que, ao mesmo tempo, apresentam o funcionamento do sistema POLCA e mostram sua aplicação na prática. Krishnamurthy e Suri (2009) fornecem uma visão geral do sistema POLCA, bem como apresentam um procedimento detalhado para a execução desta ferramenta em uma fábrica, utilizando uma abordagem de questões práticas. Dessa maneira, foi possível apresentar a aplicação do procedimento por meio de estudos de casos em três empresas diferentes. Os resultados obtidos indicaram que o sistema POLCA ajudou significativamente estas empresas a melhorarem a eficiência de suas operações, incluindo reduções de lead time, aumento na porcentagem de entregas no prazo e satisfação de seus funcionários. Riezebos (2010) faz uma investigação e posterior discussão do sistema POLCA por meio de uma revisão da literatura que apresenta os métodos e as ferramentas utilizados. Depois da revisão, o autor apresenta uma proposta de implementação do sistema em uma empresa, em que foi aplicada por meio de um estudo de caso. Os resultados concluíram que o sistema POLCA é capaz de controlar processos complexos, com grande quantidade de rotas, mix de produto e variedade no tempo de processamento. Outros benefícios também são citados como o aumento da satisfação dos funcionários, aumento na confiabilidade e melhorias no controle.

Por fim, tem-se um trabalho que trata conjuntamente de três objetivos: apresenta o funcionamento do sistema POLCA, compara qualitativamente o POLCA com outros SCOs e demonstra sua aplicação na prática. Neste trabalho, Suri e Krishnamurthy (2003) apresentam uma visão geral sobre o sistema POLCA, além de explicar seu funcionamento e fornecer comparações qualitativas com os sistemas empurrado/MRP e puxado/Kanban. Posteriormente, é apresentado um procedimento passo a passo para a implementação do POLCA em uma fábrica, bem como sua implementação, que é demonstrada por meio de dois estudos de caso. Os resultados mostraram que a aplicação do sistema POLCA trouxe algumas melhorias para estas empresas como a redução de lead time e WIP, $\mathrm{o}$ aumento na porcentagem de entregas no prazo e o aumento da satisfação do empregado.

\subsection{Trabalhos que consideram o sistema POLCA modificado}

Nesta categoria, encontram-se apenas três artigos. Um deles apresenta uma combinação do funcionamento do sistema POLCA modificado e uma comparação quantitativa com outros SCOs (seção 4.2.1); e dois representam o funcionamento do sistema POLCA modificado em conjunto com sua aplicação na prática (seção 4.2.2).

\subsubsection{Trabalhos que apresentam o funcionamento do sistema POLCA modificado e uma comparação quantitativa com outros SCOs}

No trabalho de Fernandes e do Carmo-Silva (2006), é apresentado o GPOLCA (Generic POLCA), uma adaptação do sistema POLCA que implementa uma estratégia de liberação de ordens com controle dos inputs-outputs baseado num inventário de cartões de autorização de produção, em vez de materiais. De acordo com os autores, essa estratégia é mais adequada para as empresas que têm uma grande variedade de produtos com demanda variável. O trabalho apresenta o mecanismo e as propriedades do GPOLCA, além de uma comparação de seu desempenho com o POLCA e o Kanban, realizado por meio de simulação. Os resultados mostram que o GPOLCA apresentou-se mais robusto perante as alterações no mix de produtos. Os benefícios do GPOLCA são atribuídos ao fato de que é um mecanismo com controle natural de fluxo de material empurrado e de que a manutenção de um inventário de cartões de autorização de produção, em vez de um inventário de materiais, parece ter um efeito significativo sobre a redução do WIP, principalmente para altos níveis de taxa de transferência.

\subsubsection{Trabalhos que apresentam o funcionamento do sistema POLCA modificado e mostram sua aplicação na prática}

Nesta categoria, tem-se dois trabalhos com estudos muitos semelhantes, uma vez que o segundo representa um aprimoramento do primeiro. Em Vandaele et al. (2004), é apresentada a proposta de um modelo modificado do sistema POLCA, que combina as vantagens dos sistemas empurrado e puxado. O modelo denominado E-POLCA é uma versão baseada na programação de carga do POLCA utilizado para determinar a liberação de autorizações e a carga de trabalho permitida de acordo com um sistema Advanced Resources Planning (ARP). Além disso, o trabalho descreve a implementação desta nova proposta em uma empresa que produz componentes e sistemas de transmissão para automóveis fora de estrada. Os resultados mostraram que o modelo oferece 
vantagens significativas comparadas com um sistema de programação finita e resulta em um sistema que gerencia os fluxos de materiais necessários por meio dos recursos disponíveis que têm a maior probabilidade de estarem disponíveis. Como continuação deste trabalho, Vandaele et al. (2008) propõem o Load-based POLCA (LB-POLCA), uma versão modificada do POLCA, baseada na programação de carga em um ambiente job shop com múltiplos produtos. Esta nova versão determina os parâmetros POLCA de acordo com um sistema ARP, que capta adequadamente o comportamento estocástico do sistema de produção e permite ajustes finos e um alto nível de otimização do tamanho de lote de fabricação. Posteriormente, é discutida a implementação de um sistema LB-POLCA na mesma empresa citada anteriormente. Os resultados preliminares indicaram que o sistema de LB-POLCA oferece vantagens comparadas com um sistema de programação finita, uma vez que é um sistema de autorregulação, muito mais simples de executar e mais robusto. A força da abordagem está na integração do LB-POLCA com um sistema ARP, contribuindo de forma vantajosa na prática industrial.

\section{Análises}

Nesta seção, é apresentada uma análise geral da revisão bibliográfica, utilizando-se o sistema de classificação proposto, a fim de proporcionar melhor entendimento do que a literatura oferece atualmente sobre o sistema POLCA. Dessa forma, a análise se baseia em dois pontos fundamentais: i) estudo quantitativo a respeito das quatro categorias do sistema de classificação proposto; e ii) estudo qualitativo dos principais assuntos e objetivos alcançados por esses trabalhos.

\subsection{Análise quantitativa}

A primeira análise (Figura 2) é referente ao sistema POLCA considerado pelos trabalhos estudados. Dos 20 trabalhos encontrados, 17 (85\%) utilizaram o POLCA original introduzido por Suri (1998) e apenas $3(15 \%)$ adotaram uma modificação do POLCA, ou seja, uma nova versão desse sistema. Tem-se ainda que essas versões modificadas (E-POLCA, GPOLCA e LB-POLCA) foram propostas nos últimos anos, respectivamente 2004, 2006 e 2008.

Com relação ao objetivo principal da pesquisa, tem-se que 9 trabalhos apresentam o funcionamento do POLCA $(47,4 \%) ; 5$ fazem uma comparação qualitativa do POLCA com outros SCOs (26,3\%); 5 apresentam uma comparação quantitativa do POLCA com outros SCOs (26,3\%); 5 mostram uma aplicação prática do sistema POLCA (26,3\%); 3 mostram uma simulação prática do sistema POLCA $(15,8 \%)$; e 2 apresentam o sistema POLCA como alternativa para o controle da produção do sistema produtivo (10,5\%). A Figura 3 mostra tais dados. Uma observação importante a respeito das porcentagens é que a soma delas ultrapassa $100 \%$, pois há trabalhos que foram classificados com mais de um objetivo principal. É interessante notar que o número de trabalhos teóricos é ainda muito maior do que o número de trabalhos que apresentam o sistema POLCA na prática. Esse é, com certeza, uma área interessante para estudos futuros.

Em se tratando da fonte de publicação dos trabalhos, tem-se que 10 artigos foram publicados em periódicos internacionais (50\%); 7 artigos foram apresentados em congressos locais (35\%); 1 , apresentado em congresso internacional (5\%); 1, em congresso nacional (5\%); e 1 é referente a livro (5\%). Observou-se que não há nenhuma publicação em periódico nacional (Figura 4).

Por fim, a Figura 5 mostra a distribuição de trabalhos de acordo com a metodologia de pesquisa utilizada: 11 utilizam uma metodologia teóricoconceitual $(64,7 \%) ; 7$, simulação $(41,2 \%)$; 5, estudo de caso $(29,4 \%)$; e somente 1 utiliza modelo de filas $(5,9 \%)$. Esta categoria também contém trabalhos classificados com mais de uma metodologia utilizada, resultando em uma soma das porcentagens superior a $100 \%$.

\subsection{Análise qualitativa}

Esta análise baseia-se na revisão bibliográfica apresentada na seção 4 e tem como objetivo dar suporte para maior entendimento da literatura existente

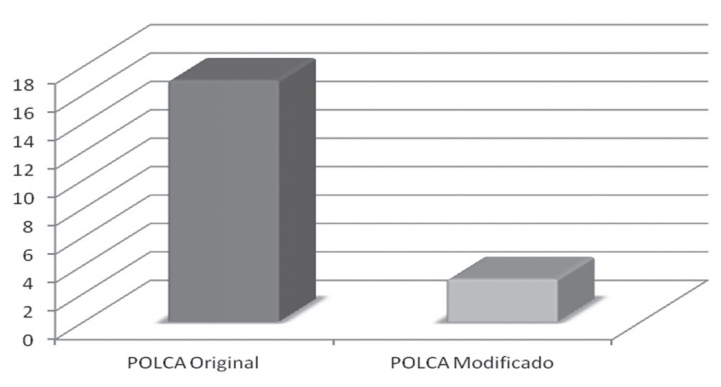

Figura 2. Número de artigos de acordo com o sistema POLCA considerado.
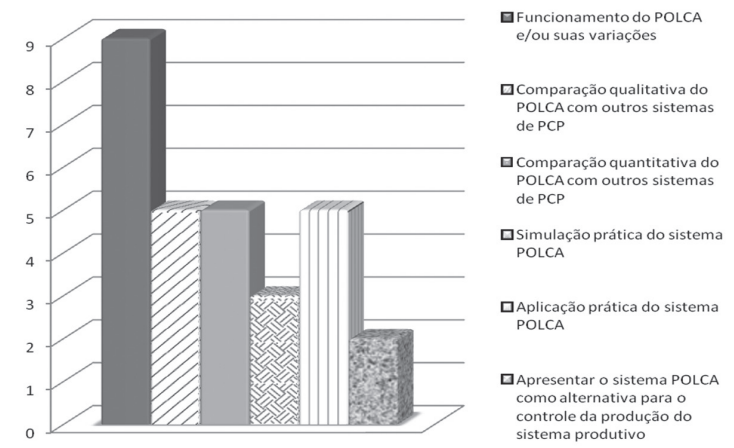

Figura 3. Número de artigos relacionados ao objetivo principal da pesquisa. 


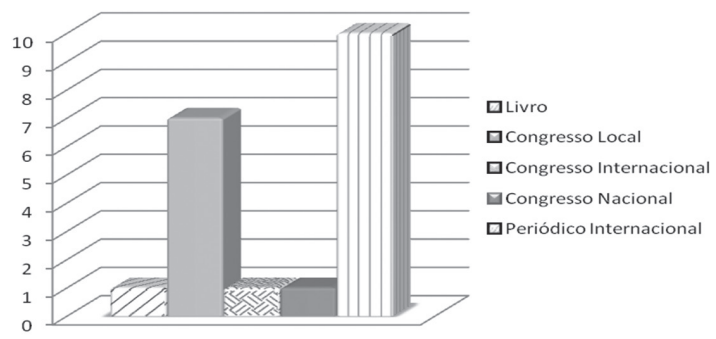

Figura 4. Número de artigos de acordo com a fonte de publicação.

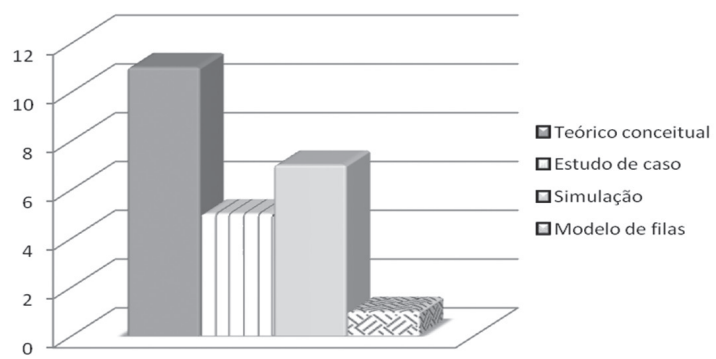

Figura 5. Número de artigos de acordo com a metodologia de pesquisa.

sobre o sistema POLCA, assim como sugerir novas pesquisas na área. Os pontos mais importantes a serem destacados são:

- Ainda são poucos os trabalhos que estudaram um sistema modificado do POLCA, como pode ser visto em Fernandes e do Carmo-Silva (2006), que apresentou o GPOLCA, e em Vandaele et al. (2004, 2008), que apresentaram o E-POLCA e LB-POLCA, respectivamente. As diferenças entre esses sistemas e o POLCA original consistem, no caso do GPOLCA, em uma liberação de ordens com controle dos inputs-outputs baseado num inventário de cartões de autorização de produção. Já no E-POLCA e LB-POLCA, a liberação de autorizações e a carga de trabalho são programadas de acordo com um sistema ARP, porém com a distinção de que no LB-POLCA a programação de carga é baseada em um ambiente job shop com múltiplos produtos.

- Muitos autores utilizam métodos de comparação qualitativos ou quantitativos a fim de mostrar diferenças/semelhanças e vantagens/desvantagens entre o POLCA e outros sistemas de PCP, tais como: Kanban, MRP, TOC, WLC, CONWIP, m-CONWIP, DEWIP, LOOR. Exemplos destes trabalhos são: Krishnamurthy, Suri e Vernon (2000), Suri (2003), Suri e Krishnamurthy (2003), Lödding, Yu e Wiendahl (2003), Fernandes, Godinho Filho e Fonseca (2005), Stevenson, Hendry e Kingsman (2005), Suri

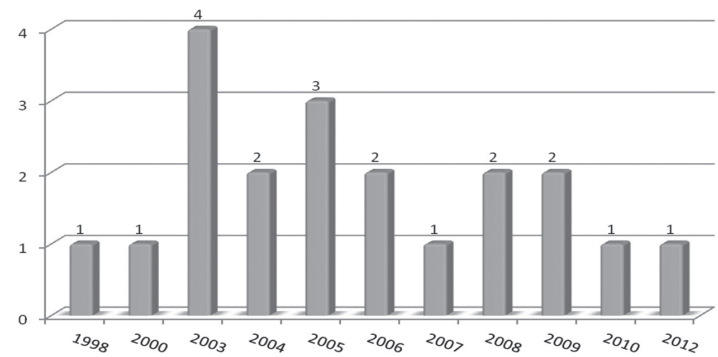

Figura 6. Evolução no tempo quanto ao número de artigos publicados.

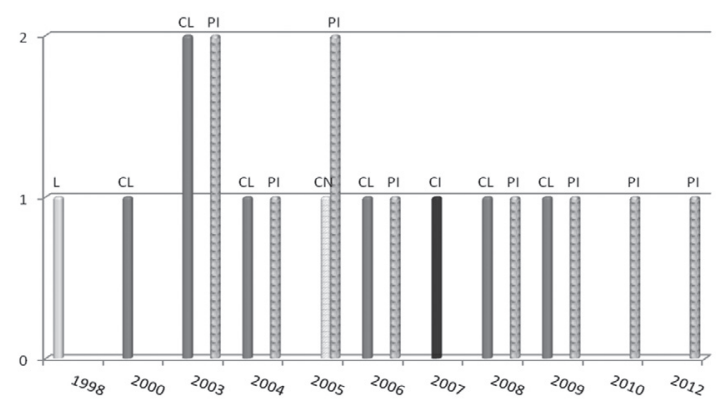

Figura 7. Evolução no tempo por tipo de publicação.

(2005), Fernandes e do Carmo-Silva (2006), Germs e Riezebos (2008) e Kabadurmus (2009).

- Atualmente, a simulação vem sendo utilizada por alguns autores para quantificar e justificar a implantação do sistema POLCA nas empresas, como foi mostrado por Riezebos (2006), Baysan, Kabadurmus e Durmusoglu (2007) e Harrod e Kanet (2013).

- Alguns poucos estudos apresentam a aplicação na prática do sistema POLCA: Suri e Krishnamurthy (2003), Vandaele et al. (2004, 2008), Krishnamurthy e Suri (2009) e Riezebos (2010). Esses estudos de caso demonstram que o sistema POLCA vem despertando interesse em empresas que desejam reduzir seu lead time em um ambiente de alta variedade de produtos.

- Em relação à fonte de publicação, é importante ressaltar que apenas um trabalho estudado é de origem nacional, uma vez que o sistema POLCA é ainda pouco conhecido e utilizado no Brasil.

- Ao considerar a evolução do número de artigos publicados desde 1998, quando Suri apresentou o sistema POLCA pela primeira vez, pode-se perceber que a partir de 2003 houve um aumento significativo de artigos publicados principalmente em periódicos internacionais e congressos locais (Figuras 6 e 7). Grande parte deste crescimento é resultado da implantação do sistema POLCA em empresas que decidiram adotar este sistema como parte das estratégias do QRM na manufatura. 


\section{Conclusões}

POLCA (Paired-cell Overlapping Loops of Cards with Authorisation) é um sistema de coordenação de ordem (SCO) introduzido por Suri (1998) como parte de uma estratégia global do QRM (Quick Response Manufacturing). De acordo com Suri (1998), é uma estratégia híbrida, que combina características do MRP e do Kanban, capaz de contornar as limitações de SCOs já existentes, em ambientes de alta variedade, demonstrando, assim, a sua relevância no setor de produção sob encomenda.

O presente trabalho, em primeiro lugar, teve por objetivo realizar uma ampla revisão bibliográfica sobre POLCA. A partir desta revisão, pôde-se propor um sistema de classificação sobre o tema com base em quatro parâmetros: sistema POLCA considerado (original/modificado), objetivo principal da pesquisa, fonte da publicação e metodologia de pesquisa empregada. Utilizando este sistema, foram classificados todos os artigos encontrados na literatura. Esse procedimento serviu de base para a estruturação de toda a revisão bibliográfica e para uma análise (quantitativa e qualitativa) do tema. Algumas conclusões interessantes a respeito do assunto e que podem auxiliar tanto a teoria quanto a prática referente ao tema são destacadas a seguir.

Referente à teoria a respeito do sistema POLCA, tem-se que existe muito espaço para a proposta de novas melhorias no sistema POLCA original, ou mesmo a hibridização de tal sistema com outro SCO, uma vez que somente 3 variações do POLCA foram encontradas na literatura. Além disso, parece existir uma grande lacuna na comparação, via simulação ou teoria de filas, do sistema POLCA a outros SCOs utilizados em ambientes de alta variedade de produtos, tais como o sistema MRP, WLC, entre outros. Somente poucos trabalhos realizam tais comparações. Finalmente, pode-se dizer que existe um grande espaço na literatura para mostrar a aplicação do sistema POLCA na prática, uma vez que casos práticos de tal sistema ainda são raros na literatura.

Referente à prática, principalmente brasileira, tem-se que o POLCA ainda é pouco conhecido e estudado em território nacional. Devido a esse fato, muitas oportunidades existem para os gerentes de Planejamento e Controle da Produção na prática, uma vez que internacionalmente tal sistema vem mostrando excelentes resultados em termos de redução de lead time.

A partir dos resultados apresentados, acredita-se que o presente trabalho contribuiu da seguinte forma: i) aumentar o grau de conhecimento e divulgação do sistema POLCA no Brasil; ii) apresentar aos gerentes de produção uma alternativa/complementação aos sistemas MRPs para empresas que enfrentam ambientes com alta variedade e alta complexidade no fluxo de materiais.

\section{Agradecimentos}

Os autores gostariam de agradecer a FAPESP e CNPQ por auxiliarem o presente trabalho.

\section{Referências}

BAYSAN, S.; KABADURMUS, O.; DURMUSOGLU, M. B. Economic Analysis of POLCA System Design via Simulation: A Multi-Cell Manufacturing Case Study. In: INTERNATIONAL CONFERENCE ON COMPUTERS AND INDUSTRIAL ENGINEERING, 37., 2007, Alexandria, Egypt. Proceedings... Alexandria, 2007.

BERTO, R. M. V. S.; NAKANO, D. N. A produção científica nos anais do encontro nacional de Engenharia de Produção: um levantamento dos métodos e tipos de pesquisa. Produção, v. 9, n. 2, p. 65-75, 2000. http:// dx.doi.org/10.1590/S0103-65131999000200005

FERNANDES, F. C. F.; GODINHO FILHO, M.; FONSECA, R. A. Discussão a respeito do funcionamento, das características e da aplicabilidade de três recentes sistemas de Coordenação de Ordens: DEWIP, LOOR e POLCA. In: ENCONTRO NACIONAL DE ENGENHARIA DE PRODUÇÃO, 25., 2005, Porto Alegre. Anais... Porto Alegre, 2005.

FERNANDES, N. O.; DO CARMO-SILVA, S. Generic POLCAa production and materials flow control mechanism for quick response manufacturing. International Journal of Production Economics, v. 104, n. 1, p. 74-84, 2006. http://dx.doi.org/10.1016/j. ijpe.2005.07.003

FERNANDES, F. C. F.; GODINHO FILHO, M. Sistemas de coordenação de ordens: classificação, funcionamento e aplicabilidade. Gestão \& Produção, v. 14, n. 2, p. 337-352, 2007. http://dx.doi.org/10.1590/ S0104-530X2007000200011

FERNANDES, F. C. F.; GODINHO FILHO, M. Planejamento e controle da produção: dos fundamentos ao essencial. São Paulo: Atlas, 2010.

FILIPPINI, R. Operations management research: some reflections on evolution, models and empirical studies in OM. International Journal of Operations and Production Management, v. 17, n. 7, p. 655-70, 1997. http://dx.doi.org/10.1108/01443579710175583

GERMS, R.; RIEZEBOS, J. Workload balancing capability of pull systems in MTO Production. In: INTERNATIONAL WORKING SEMINAR ON PRODUCTION ECONOMICS, 15., 2008, Innsbruck. Preprints... Innsbruck, 2008. p. 217-228.

GOOD, I. J. Categorisation of classification. In: BARTHOLOMAY, A. F. Mathematics and computer science in medicine and biology. London: HMSO, 1965. p. 115-128.

HARROD, J.; KANET, J. J. Applying work flow control in make-to-order job shops. International Journal of Production Economics, v. 143, n. 2, p. 620-626, 2013. http://dx.doi.org/10.1016/j.ijpe.2012.02.017

KABADURMUS, O. A comparative study of POLCA and generic CONWIP production control systems in erratic demand conditions. Auburn University, 2009. IIE Lean Division - Lean Student Paper Competition. 
KRISHNAMURTHY, A.; SURI, R. Planning and implementing POLCA: a card-based control system for high variety or custom engineered products. Production Planning \& Control, v. 20, n. 7, p. 596-610, 2009. http://dx.doi.org/10.1080/09537280903034297

KRISHNAMURTHY, A.; SURI, R.; VERNON, M. K. A new approach for analyzing queueing models of material control strategies in manufacturing systems. In: INTERNATIONAL WORKSHOP ON QUEUEING NETWORKS WITH FINITE CAPACITY, 4., 2000, Ilkley, West Yorkshire. Proceedings... Ilkley, 2000. p. 1-26.

KRISHNAMURTHY, A.; SURI, R.; VERNON, M. K. Re-examining the performance of MRP and KANBAN material control strategies for multi-product flexible manufacturing systems. International Journal of Flexible Manufacturing Systems, v. 16, n. 2, p. 123-150, 2004. http://dx.doi.org/10.1023/B:FLEX.0000044837.86194.19

LÖDDING, H.; YU, K. W.; WIENDAHL, H. P. Decentralized WIP-oriented manufacturing control (DEWIP). Production Planning and Control, v. 14, n. 1, p. 42-54, 2003. http://dx.doi. org/10.1080/0953728021000078701

NAKANO, D. Métodos de pesquisa adotados na Engenharia de Produção e Gestão de Operações. In: MIGUEL, P. A.M (Org.). Metodologia de pesquisa em engenharia de produção e gestão de operações. Rio de Janeiro: Elsevier, 2010, p. 63-72.

NGAI, E. W. T. et al. RFID research: An academic literature review (1995-2005) and future research directions. International Journal of Production Economics, v. 112, n. 2, p. 510-520, 2008. http://dx.doi.org/10.1016/j. ijpe.2007.05.004

RIEZEBOS, J. Polca simulation of a unidirectional flow system. In: RIEZEBOS, J.; SLOMP, J. Proceedings of the third international conference on group technology/cellular manufacturing. Groningen: University of Groningen, 2006. p. 332-338.

RIEZEBOS, J. Design of Polca material control systems. Internacional Journal of Production Research, v. 48, n. 5, p. 1455-1477, 2010. http://dx.doi. org/10.1080/00207540802570677
ROWLEY, J.; SLACK, F. Conducting a literature review. Management Research News, v. 27, n. 6, p. 31-39, 2004. http://dx.doi.org/10.1108/01409170410784185

RYAN, C. M.; CHOOBINEH, F. Total WIP and WIP mix for a CONWIP controlled job shop. IIE Transactions, v. 35, n. 5, p. 405-418, 2003. http:// dx.doi.org/10.1080/07408170304387

SHIMIZU, T. Pesquisa operacional em engenharia, economia e administração: modelos básicos e métodos computacionais. Rio de Janeiro: Guanabara Dois, 1984.

STEVENSON, M.; HENDRY, L. C.; KINGSMAN, B. G. A review of production planning and control: the applicability of key concepts to the make-to-order industry. International Journal of Production Research, v. 43, n. 1, p. 869-898, 2005. http://dx.doi. org/10.1080/0020754042000298520

SURI, R. Quick response manufacturing: a companywide approach to reducing lead times. Portland: Productivity Press, 1998.

SURI, R. QRM and POLCA: a winning combination for manufacturing enterprises in the 21st century. Madison: University of Wisconsin-Madison, 2003. Technical Report Center for Quick Response Manufacturing.

SURI, R. A lean strategy for job shops. Gear Technology. Journal of Gear Manufacturing, Nov-Dec. 2005.

SURI, R. It's about time: the competitive advantage of quick response manufacturing. New York: Productivity Press, 2010. http://dx.doi.org/10.1201/EBK1439805954

SURI, R.; KRISHNAMURTHY, A. How to plan and implement POLCA: a material control system for high-variety or custom-engineered products. Madison: University of Wisconsin-Madison, 2003. Technical Report Center for Quick Response Manufacturing

VANDAELE, N. et al. E-POLCA to control multi-product, multimachine job shops. Working paper University of Antwerp, 2004.

VANDAELE, N. et al. Load-based POLCA: an integrated material control system for multiproduct, multimachine job shops. Manufacturing \& Service Operations Management, v. 10, n. 2, p. 181-197, 2008. http:// dx.doi.org/10.1287/msom.1070.0174 